\title{
LOS IULII EN EUROPA CENTRAL: UN GRUPO GENTILICIO ROMANO A TRA- VES DE LAS FUENTES EPIGRAFICAS
}

\author{
HENAR GALLEGO FRANCO \\ Universidad de Valladolid
}

\begin{abstract}
En el conjunto de las fuentes epigráficas provinciales de Rhaetia y Noricum, los lulii resultan ser el grupo gentilicio de mayor presencia individual en estas provincias. Realizamos un análisis onomástico y social de esta familia en base a la documentación epigráfica, señalando las características de sus esquemas antroponímicos básicos, así como sus vías de contribución e integración en las estructuras socioeconómicas y religiosas provinciales, estableciendo, en última instancia, su papel en el mapa onomástico y de romanización de la zona.
\end{abstract}

According to the epigraphical sources of Rhaetia and Noricum, the lulii appear as the main family group in these roman provinces. We carry out an onomastic and social study of this family on the basis of the epigraphical information, looking for the features most important characteristics of their personal names and their way of contribution to the socioeconomic and religious provincial structures. Finally, we establish the role of the lulii in the onomastic map and the romanization process of these provinces.

Los Alpes centrales y el territorio que se extiende al norte, donde se asentarán luego las provincias romanas de Rhaetia y Noricum en Europa Central, atraen desde un momento temprano el interés romano. Su ámbito geográfico viene a coincidir, en líneas generales, con la zona sur de Alemania (la actual Baviera), Austria, y noroeste de la antigua Yugoslavia, alrededor de las ciudades eslovenas de Celje y Maribor. Este área controlaba los accesos y puntos de paso de numerosas rutas de comercio de dirección norte-sur desde Italia, las que iban hacia Germania y Gallia (centro-oeste), y las que llegaban hasta las riberas del Danubio, donde se encontraban con las rutas que venían del mar Báltico, como la del ámbar. En este sentido, la presencia del limes y la existencia de un curso fluvial de primer orden como es el Danubio, tuvo el efecto altamente estimulador de las actividades de intercambio en las provincias del alto y medio curso del río, objeto de este estudio ${ }^{1}$. También tenía gran interés el comercio con los pueblos fronterizos, como Quados y Marcomanos, que era utilizado por Roma como un mecanismo de control sobre estas tribus
(WHITTAKER, 1989, 59-73; DRUMMOND Y NELSON, 1994, 101-126). Asi, el territorio de Retii y Vindelici, y el antiguo reino celta de Noricum, entraron en contacto de forma temprana y pacífica con Roma a través de los mercaderes itálicos y sus agentes, que penetraron en la región atraídos por sus posibilidades comerciales y su extraordinaria riqueza minera, especialmente por las minas de hierro noricanas (WACHER, 1987, 161-170; EGGER, 1961; DUSANIC, 1977, 52-94), siendo finalmente anexionado y convertido en provincias romanas a finales del s.l a.C. ${ }^{2}$.

El estudio onomástico de los ciudadanos de Rhaetia y Noricum portadores de un nomen gentilicio, basado en las fuentes epigráficas pro-

\footnotetext{
${ }^{1}$ Cfr., entre otras, CHARLESWORTH, 1976 (Ed. 1926); SGHLIPPSCHUH, 1974; D'ARMS, 1981; WALSER, 1983; GARNSEY, HOPKINS Y WHITTAKER, 1983; WHITTAKER, 1989; WHITTAKER, 1994; DRUMMOND Y NELSON, 1994

${ }^{2}$ Dion Cassio, LIV, 20-22; Velleio Patérculo, Hist. Rom., 11, 95; Floro, Epitome, II, 22; Plinio el Viejo, Nat. Hist, III, 136; WAĊHER, 1987, 224-225; ULBERT, 1971, 101-123; MARTIN, 1990, 95-98; KELLNER, 1976, 690-717.
} 
vinciales, revela que un grupo selecto de familias gentilicias se distingue por su destacada contribución cuantitativa, la cual se traduce, además, en una participación privilegiada de sus miembros en las estructuras sociales provinciales. Éstos manifiestan una actividad pública y social más rica y variada. En ellos las élites urbanas están mejor constatadas, acceden más frecuentemente al ejercicio de magistraturas y cargos religiosos, forman parte de las asociaciones y collegia, a menudo ejercen como patronos de libertos y dueños de esclavos, símbolo de la buena posición socioeconómica de parte de sus miembros, y participan más activamente en el fenómeno votivo-religioso (GALLEGO, 1997, 104-157).

Este grupo de grandes familias supone aproximadamente un tercio del total de individuos de Noricum integrados en grupos gentilicios, mientras que en Rhaetia la proporción que aportan aumenta hasta casi el $50 \%$. En este conjunto de grandes familias, monopolizado por los nomina imperiales, los protagonistas indudables son los Iulii, seguidos de los Aurelii, Claudii, Flavii, Aelii y Ulpii. En definitiva, la distinta incidencia cuantitativa de los grupos o familias gentilicias en el mapa onomástico provincial genera una secuencia romanizadora, poniendo de manifiesto las diferentes etapas del proceso de asimilación de las estructuras romanas, así como su desigual importancia y el distinto peso de la política de los emperadores en la difusión de los derechos de ciudadanía, uno de cuyos canales principales sigue siendo el servicio en el ejército. Las grandes familias traducen la huella de la política romanizadora de los diferentes emperadores y dinastías en el alto y medio Danubio, aunque tampoco podemos olvidar la aportación de los desplazamientos de población de otros puntos del Imperio hacia Rhaetia y Noricum, especialmente de Italia y las provincias occidentales (GALLEGO, 1997, 132133, 154-157).

En el presente trabajo abordamos un estudio del grupo gentilicio de los $l u l i i^{3}$ a través de los testimonios recogidos en las fuentes epigráficas de Rhaetia y Noricum ${ }^{4}$. Nuestro análisis se centrará en primer lugar en las características onomásticas de la familia, desentrañando las claves básicas y peculiaridades de los esquemas antroponímicos de sus miembros, para realizar, en un segundo momento, un estudio las formas y mecanismos de participación del grupo gentilicio en las estructuras socioeconómicas, administrativas y religiosas de las provincias romanas de Rhaetia y Noricum.
La presencia de testimonios masculinos en este grupo gentilicio es claramente mayoritaria, de manera que los hombres aportan las tres cuartas partes del total de lulii en Rhaetia y algo más de las dos cuartas partes en Noricum. El análisis onomástico de estos varones manifiesta una tendencia a la superioridad del duo nomina, de proporciones desiguales en cada provincia. En Rhaetia el triunfo del duo nomina es casi aplastante, extendiéndose a más de las tres cuartas partes de los varones. En cambio los esquemas de duo y tria nomina aparecen casi igualados en Noricum. Incluso la onomástica masculina de Noricum muestra que la incidencia del praenomen es incluso ligeramente superior a la del tria nomina, lo que evidencia que este esquema antroponímico pudo alcanzar un portentaje más elevado del conservado. De hecho algunos individuos con praenomen y nomen no mencionan su cognomen, bien por omisión (filiaciones, patronos de libertos, marcas de cerámica) o simplemente porque éste no se ha conservado en el documento epigráfico. Esta omisión o pérdida del cognomen también existe en algunos individuos de los que conservamos sólo el gentilicio, y que debieron de portar, sin duda, su cognomen en un esquema de duo nomina. El nombre único entre los /ulii masculinos de Noricum aparece en un solo caso, y por influencia de una onomástica prerromana, cuyo sistema era el de un nombre seguido del patronímico: lulius 4 Secundi f(ilius) (CIL III 5196).

En todo caso, la explicación de esta distribución del praenomen entre los varones parece ser cronológica, perteneciendo mayoritariamente los individuos que lo usan a los dos primeros siglos del Imperio. Sin embargo, si comparamos los lulii noricanos que portan duo nomina con los que portan tria nomina en los dos primeros siglos del Imperio, encontramos que pese a ser mayoría los primeros, el mayor porcentaje de cargos militares y civiles se concentra en el segundo grupo. Por tanto el uso del

\footnotetext{
${ }^{3}$ SOLIN Y SALOMIES,1988,98; $\mathrm{PIR}^{2}$ | 192-710; GALLEGO, 1996, 120-131, 264-284, los luliisuponen en Rhaetia el 16\% de los individuos portadores de nombre gentilicio, y en Noricum el $10 \%$.

${ }^{4}$ La bibliografía referente a fuentes epigráficas aparece en el presente trabajo atendiendo a las siguientes abreviaturas: AE (ANNEE EPIGRAPHIQUE, 1900-1992); AL (ALFOLDY, 1969, 1-34); CIL (CORPUS INSCRIPTIONUM LATINARUM, III y suppl.); EE (HENZEN, ROSSI, MOMMSEM Y WILMANNS, II, 1875, IV, 1881); IBR (VOLLMER, 1915); ILI (SASEL, 1963; SASEL, 1978; SASEL, 1986); IRS (WEBER, 1969); NIR (WAGNER, 1956-1957 [1958], 215-264).
} 
tria nomina, y especialmente la mención del praenomen en Noricum, responde a cuestiones cronológicas, pero también sociales, teniendo más incidencia entre aquellos individuos que por su posición social pueden estar en disposición, de alguna manera, de representar lo que es el Estado romano y su forma de vida.

En consecuencia, en Rhaetia el praenomen aparece minoritariamente, siendo muy frecuente el de $C$ (aius). Precisamente la asociación $C$. lulius es la de César, será la de Augusto tras su adopción por éste, y la de otros miembros de la gens lulia imperial, como Calígula. También existe la asociación Ti(berius) lulius, que hace referencia al emperador Tiberio, o la de $T$ (itus) lulius, que alude a los Antoninos, probablemente a Hadriano. El uso de la tribu, sin embargo, es muy escaso entre los varones de Rhaetia. Contamos con un solo caso, el de P. lulius Pintamus (AE72, 359), un hispano de Bracara Augusta (Braga, Portugal), que pertenece a la tribu Quirina, y que así lo hace constar en su epígrafe funerario de Leutstteten, cerca de Augsburg (Bayern, Alemania). La práctica ausencia de la tribu y el uso reducido del praenomina se explican sin duda por la adscripción cronológica de la mayoría de los /ulii réticos al período que abarca la segunda mitad del s.II y el s.III d.C.. La mención de la tribu en la onomástica es rara a partir de Trajano y Hadriano, y la del praenomen escasea desde la segunda mitad del s.ll para caer en desuso en el s.lll. Sin embargo es significativo que el único caso en el que se cita la tribu es en la onomástica de un extranjero en la provincia. Evidentemente la tribu era más proclive a ser mencionada en relación con la origo, formando un bloque de rasgos distintivos del individuo.

Entre los lulii réticos contamos con tres extranjeros que expresan su origo. Uno de ellos, como ya indicamos anteriormente, es $P$. lulius Pintamus (AE72, 359), domo ex Hispania Citeriore, Augusta Bracara, veteranus ex decurione alae..., decurio municipii Aelii. Se trata, como vemos por su cognomen celta (HOLDER, II, 1962, 1005), de un indígena romanizado que sirvió en el ejército en un cuerpo auxiliar, y conseguida la ciudadanía, llegó a ingresar en la aristocracia municipal de Augusta Vindelicorum (Augsburg, Alemania) ${ }^{5}$, la capital, de la que fue decurio. Los otros dos son también de procedencia occidental, Iulius Dubitatus (CIL III 5936), cives rutenus (Gallia Narbonensis), y lulius Genialis (CIL III 5907), cives mediomatricus (Gallia Belgica), veteranus alae, que sirvió en un cuerpo auxiliar militar. Su origo indica igual- mente que se trata de indígenas romanizados. También lo era el liberto C. lulius Libertus (CIL III 5831), natione Biturix (Gallia Aquitania), antiguo esclavo celta.

Sin embargo, extranjeros en Rhaetia eran igualmente el legatus Augusti lulius Avitus Alexianus (AE62, 229), y los hermanos lulius Leontius y lulia Leontia (AE78, 577), cuyos cognomina griegos (SOLIN, 1982, 196, 1356, $1052,1056,1347,1364,1054,1337,1364)$ responderían a un origen greco-oriental. Los extranjeros entre los lulii réticos presentan por tanto una doble procedencia: individuos naturales de otras zonas celtas de Occidente, cuyos canales de desplazamiento han sido la esclavitud o el ejército, e individuos que proceden de la zona greco-oriental del Imperio, cuya movilidad se adscribe al mundo profesional y a su propia carrera en la administración imperial. En otros casos el cognomen griego parece responder a la moda del momento, como el que ostenta el decurio de Augusta Vindelicorum C. Iulius Achilleus (NIR 33; SOLIN, 1982, 464).

Volviendo a la provincia romana de Noricum, el praenomen más frecuente es Ti(berius) o Tib(erius), seguido a corta distancia por C(aius), lo que evidencia que un grupo importante de los Iulii de Noricum accedieron a la ciudadanía romana bajo los reinados de Augusto y Tiberio. Testimonio directo de ello lo tenemos en la persona de C. Iulius Vepo (CIL III 5232; SASEL, 1954, 346-363), donatus civitate romana viritim et inmunitate ab divo Augusto en Celeia (Celje, Eslovenia) ${ }^{6}$. Los otros praenomina constatados son T(itus), L(ucius), M(arcus) y Sex(tus), todos ellos de forma minoritaria. Algunos pueden tener su origen en la onomástica imperial, como L(ucius), que era el praenomen de L. Caesar, el hijo de Agrippa y Iulia, heredero de Augusto, que murió en el 4 d.C., o el de $T$ (itus), que es característico de la dinastía Flavia, de manera que los individuos que lo usan pertenecen a familias que accedieron a la ciudadanía con los julio-claudios, pero que usan quizá el praenomen de moda, el de la dinastía reinante, lo que coincide además en un número significativo de casos con la cronología de los epígrafes en que son constatados. Ahora bien, la causa directa del uso de un determinado praenomen también puede estar en las relaciones de dependencia entre particulares y las re-

\footnotetext{
5 Ptolomeo, 2,12,4; 8,7,4; Itin. Anton., 232,1; 236,5; 241,4; 250,$5 ; 258,2.11 ; 274,8$; Not. Dign. Occ., 11,30 .

6 Plinio, N.H., 3,146; Ptolomeo, 2,13,3; Itin. Ant., 129, 4.
} 
des de influencia de las familias de notables o personajes influyentes, como por ejemplo, gobernadores provinciales.

Al igual que ocurre en Rhaetia, la mención de la tribu es muy escasa en Noricum, y, en todo caso, se vincula a una datación temprana (primera mitad s.I d.C.) y a un origen itálico, como parece ser el de los speculatores Caesaris Augusti C. Iulius Bassus 2 y L. Iulius Praesens, ambos de la tribu Velina (CIL III 4843), que servían en una unidad militar como exploradores o espías. En cambio, no se hace mención de la origo en el sistema onomástico. Ello no quiere decir que entre los lulii de Noricum no existieran extranjeros, pero son difíciles de identificar. Los cognomina griegos, asociados casi en su totalidad a antiguos esclavos, no implican necesariamente una procedencia oriental. El ejército sería también un canal de entrada de nuevos individuos en la provincia, pero hay que tener en cuenta que con el tiempo el reclutamiento tiende a hacerse local, proceso que se inicia con Vespasiano y acaba de configurarse con Hadriano ${ }^{7}$.

Tanto en Rhaetia como en Noricum el sector femenino de los lulii ofrece mayoritariamente un sistema onomástico tradicional de nomen + cognomen, lo que indica un alto índice de romanización. La desaparición del cognomen en algunos casos suele responder a un deterioro en el documento epigráfico. En otros casos femeninos de Noricum observamos que se omite por razones de familiaridad. Así a lulia 7 Titi lib(erta) (CIL III 5644) se la consideraría perfectamente identificada con la mención de su patrono. En lulia 12 (AE77, 597) la omisión del cognomen parece responder a un contexto coloquial, ya que ella y sus hijos son nombrados por un solo nombre, probablemente el que les era familiar, frente al duo nomina del cabeza de familia, Aelius Ingenus (AE77, 597). En otros casos la presencia del nombre único parece deberse a la influencia de antiguos hábitos onomásticos indígenas de Noricum, como en el caso de lulia 2, luli f(ilia) (CIL III 4907).

Un miembro femenino de la gens lulia de Noricum usa un sistema onomástico de tres elementos, al incluir un praenomen, C. Iulia luliana (CIL III 4721). El uso del praenomen por las mujeres (Liber de Praenominibus, 7; KAJANTO, 1977, 147-158) se abandona a finales de la República, cuando se generaliza el del cognomen. Sin embargo la presencia del praenomen, además del cognomen, en la onomástica femenina, ha sido atestiguada en las provincias, por ejemplo en Hispania (PASTOR Y MENDOZA,
$1987, n^{\circ} 79$ ), a menudo en una fecha temprana (s.I d.C.). Esta práctica puede tener la intención de reforzar o acentuar la romanidad de la mujer que lo porta, que habría accedido recientemente al sistema onomástico romano, bien porque era una indígena romanizada o bien por un anterior origen servil. La ausencia de la mención del parentesco entre C. Iulia luliana y los individuos que la acompañan en el epígrafe votivo, C. Iulius Mercator, C. Iulius Verecundus y $C$. Iulius Secundus, unida al hecho de que parece dedicar a una divinidad indígena $(\mathrm{C} /$ bus? Augustus), apoya cualquiera de estas dos hipótesis.

Tanto los hombres como las mujeres de los lulii de Noricum usan en ocasiones un doble nomen, que en el caso de los varones puede ir precedido además por el praenomen. El doble nomen supone muy a menudo la pervivencia en el sistema onomástico del individuo de otro grupo familiar, además del paterno, que refleja el primer gentilicio. Entre los /ulii noricanos este segundo grupo familiar puede ser, en primer lugar, el de la madre, y probablemente así ocurría con Iulia Capitonia (AE88, 910) o Iulia Cassia (AE73, 392), ambas pertenecientes a un grupo social privilegiado, ya que la primera es esposa de $Q$. Sabinius, un Ilvir iure dicundo de Virunum ${ }^{8}$, la capital de Noricum, y la segunda era patrona de dos libertos, Iulius Acceptus y lulia Quinta 2 y probablemente también de su hija Accepta. También podía ocurrir a la inversa, y que el segundo nomen reflejara la onomástica del padre, como Iulius Apricius (CIL III 5671). Éste era probablemente hijo natural y lleva el gentilicio de su madre, Iulia Severio, mientras que el segundo nomen conserva quizá la huella de la onomástica paterna, ya que no coincide con el actual esposo de la madre Seccius Secundinus. En cuanto a C. lulius Censonius (CIL III 4923), era probablemente un liberto, como su esposa Iulia Privata luliae Sabinae l(iberta), y quizá su segundo nomen reflejara la onomástica del esposo de su patrona, aunque no se han conservado los datos necesarios para corroborar esta hipótesis. En todos los casos el uso de un segundo nomen elimina la mención del cognomen, lo que evidencia que el segundo gentilicio se emplea no como un rasgo onomástico perpe-

\footnotetext{
${ }^{7}$ Suetonio, Vesp., VIII; GIARDINA, 1991, 130-135; FORNI, 1953; LUTTWAK, 1976, 124-125; CARRIE, 1986, 449-488, 760-79; CHRISTOL, 1988, 169-204.

${ }^{8}$ Ptolomeo, 2, 11, 9; 2, 13, 3; Plinio, N.H., 3, 146; Itin. Ant., $276,5$.
} 
tuado a través de varias generaciones, típico más bien de familias de la aristocracia equestre y senatorial, sino como un elemento identificativo de la persona, como lo era el cognomen.

Por otro lado, en Rhaetia la práctica común es que el nomen Iulius/a se asocie a un cognomen, y los pocos casos de ausencia de éste responden a omisión o deterioro del documento. Los cognomina latinos son absolutamente predominantes entre los lulii réticos, frente al carácter muy minoritario de los indígenas celtas $(8 \%)$ y de griegos $(6 \%)$. La distribución por sexos de los individuos que usan un cognomen griego o indígena es muy equilibrada, casi al $50 \%$. Todos estos datos evidencian que los lulii de Rhaetia son un grupo de población muy romanizado, plenamente integrado en los esquemas onomásticos oficiales que distinguían al ciudadano romano, en el que la pervivencia de la onomástica indígena es poco notoria, y en todo caso afecta por igual a hombres y mujeres.

Los cognomina latinos que pertenecen a los Iulii réticos ofrecen una gran variedad. Victor (KAJANTO, 1965, 11, 18, 30bis, 57, 72, 89, 96, 98,278 ) y sus derivados Victorina y Victorianus (KAJANTO, 1965, 278) presentan una mayor frecuencia, pero en una proporción que sigue siendo minoritaria. No parece que podamos hablar de unos cognomina característicos de esta gens en Rhaetia. La aparición del doble cognomina va asociada a individuos de status social privilegiado. Ello se refleja perfectamente en el caso de G. Iulius Avitus Alexianus, senatorial y legatus Augusti pro praetore provinciae Rhaetiae (AE62, 229; $\mathrm{PIR}^{2} \mid$ 192). Su primer cognomen es latino (KAJANTO, 1965, $18,79,80,134,304$ ) y el segundo griego (SOLIN, 1982, 196, 1356). Su onomástica le emparenta además con la familia imperial de los Severos, en la que son frecuentes los cognomina Avitus y Alexianus ${ }^{9}$.

Igualmente, casi la totalidad de los lulii del Noricum acompañan su gentilicio de un cognomen, salvo los pocos casos de nombre único, y las omisiones y pérdidas que ya hemos analizado. Al igual que en Rhaetia, predominan entre los lulii noricanos los cognomina latinos, mientras que la incidencia de los cognomina indígenas $(15 \%)$ y griegos $(10 \%)$ es muy minoritaria. Los cognomina latinos se caracterizan también por la variedad, de manera que no podemos hablar de cognomina característicos de esta familia gentilicia. En cuanto a los griegos e indígenas, recaen mayoritariamente en individuos varones. Los Iulii presentan, por tanto, tanto en Rhaetia como en Noricum, una onomástica altamente romanizada, que sigue las tendencias generales del Imperio, con muy pocas excepciones.

Los cognomina derivados del nomen lulius que se registran en Rhaetia son lulianus/a (KAJANTO, 1965, 35, 148), lullinus (KAJANTO, 1965, 162; SOLIN y SALOMIES, 1988, 346) y lulinus (KAJANTO, 1965, 162), y casi en la totalidad de los casos pertenecen a individuos masculinos. Es significativo el hecho de que cuando van acompañando a un nomen, éste es el de lulius. Este dato refleja la importancia del gentilicio lulius en determinadas familias, e implica una consolidación de la fortaleza de la gens. Cuando estos cognomina derivados no van acompañando a un nomen es porque éste se ha omitido (marcas de cerámica), o bien se ha perdido por deterioro del documento. En todo caso el fenómeno de los cognomina derivados evidencia que el número de lulii real era superior al que las fuentes han conservado, y los cognomina lulianus/a, lullinus o lulinus son pruebas indirectas de este hecho en Rhaetia.

También en Noricum la presencia de cognomina derivados del nomen lulius refuerza la posición de los lulii como el principal grupo gentilicio provincial. Estos cognomina son Iulianus/ a o lulianna (KAJANTO, 1965, 35, 148). Cuando estos cognomina derivados van acompañados de un gentilicio, muestran la vinculación de los lulii a familias importantes, bien a nivel provincial o a nivel local. Entre los primeros destaca C. Antonius Iulianus (CIL III 5120), individuo de rango equestre, procurator publici portorii (PIR $^{2}$ A 845), hijo de T. lulius Saturninus ${ }^{10}$, caballero que fue conductor publici portorii Illyrici (DEVIJVER, I, 1976/77, 120; PFLAUM, I, 19601961, 174), e hijo adoptivo de C. Antonius Rufus (CIL III 5122, 14354.29/30), de rango equestre y que también desempeñó el cargo de conductor publici portorii ( $\mathrm{PIR}^{2}$ A 871). Por otro lado, las uniones de los lulii con familias notables a nivel local se reflejan en la onomástica de $C$. Atilius Iulianus (CIL III 5115), Celerius Iulianus (CIL III 5913), Q. Marius lulianus (CIL III 5254), Q. Pompeius lulianus (CIL III 5378) o L. Septimius Iulianus (CIL III 5563).

C. Atilius Iulianus (CIL III 5115) evidencia la alianza de los Iulii con los Atilii, una familia de

${ }^{9}$ AE21, 64, señala que este individuo es natural de Dalmatia; Herodiano, V, 3,3; V, 7,9 sobre su relación con los Severos.

${ }^{10}$ CIL III 4720. 
origen noritálico (CIL III 4869) que se extiende por Noricum en el s.Il, alcanzando cierta significación, y a cuyos miembros encontramos en varias magistraturas municipales (CIL III 5462, 5225). Celerius Iulianus (CIL III 5319) era miembro de una embajada o un consejo de una civitas o comunidad autóctona adscrita a Solva (Plinio, N.H., 3,146), municipio en el que hace una dedicación a luppiter Optimus Maximus, protector del Estado romano. Q. Pompeius /ulianus es el fruto de la unión de una mujer de la gens Iulia con un individuo relacionado con los Pompeii de origen noritálico de Celeia (HARDING y JACOBSEN, 1989, 227-232), siendo además la gens Pompeia una familia de significación en Noricum (GALLEGO, 1996, 365-558). L. Septimius Iulianus (CIL III 5563) y Tib. Marius lulianus (CIL III 5254), además de pertenecer a dos familias significativas de Noricum, los Uipii y los Septimii (GALLEGO, 1996, 357-558), muestran su categoría social a través de la propiedad de siervos y el patronazgo de libertos. En cuanto a Cupitia luliana (CIL III 5211), Speratia luliana (CIL III 5240) y Ulpia luliana (AE39, 270), se trata de tres mujeres claramente libres y ciudadanas, sin ninguna sospecha de origen inferior en su onomástica y su entorno familiar.

Por otro lado, el uso de los cognomina derivados del nomen lulius entre indígenas romanizados es prueba de que el gentilicio era muy familiar en Noricum, portado por notables y ciudadanos, sinónimo del mundo romano. Los dos individuos de los /ulii noricanos que portan cognomina derivados de su nomen parecen indígenas romanizados, C. lulia luliana (CIL III 4721) y Tib. Iulius Iulianus (CIL III 4929), como puede evidenciar el praenomen femenino, y la ascendencia familiar del varón, que es hijo de Tib. Iulius Senecio y Bellicia Auteta (HOLDER, III, 1962, 764). También serían indígenas romanizados Iuliana (CIL III 5254), serva de Tib. Marius Iulianus, cuya abuela materna lleva el nombre de Curena (HOLDER, I, 1961, 1200), Iuliania Tertini f(ilia) (CIL III 5463), cuya abuela materna es Sucela Ucconis f(ilia) (HOLDER, II, 1962, 1654; HOLDER, III, 1962, 12), lulianna (CIL III 5637), esposa de Mascius lantumari f(ilius) (HOLDER, II, 1962, 9, 452), Iulio lanuae f(ilius) (HOLDER, II, 1962, 9) y su hijo Iulianus 3 (CIL III 5294), y finalmente lulianus 1 (CIL III 4999) y lulianus 2 (CIL III 5027), que son dos filiaciones, el primero la de Ursulus, esposo de Covinaerta Covinerti f(ilia) (HOLDER, I, 1961, 1152,1054 ) y el segundo la de Auno (HOLDER, I, 1961, 294).
Desde el punto de vista social, los /ulii de Rhaetia y Noricum están integrados fundamentalmente por hombres libres, los cuales participan activamente en las estructuras oficiales a diferentes niveles. Los ordines sociales superiores no están bien representados entre ellos, pero en general los grupos sociales senatoriales y equestres presentan escasa entidad en nuestras provincias (GALLEGO, 1997, 88, 159160). Entre los lulii contamos con las figuras del senatorial lulius Avitus Alexianus (AE62, 229), legatus Augusti de Rhaetia, si bien es un extranjero en la provincia, y de los equestres $T$. Iulius Saturninus (CIL III 4720), conductor publici portorii Illyrici", y quizá el militar lulius Valentinus 1 (CIL III 11772), praefectus?, ambos en Noricum. La familia también contribuye a las aristocracias municipales a través del ejercicio de cargos públicos, y así encontramos a dos /ulii como decuriones en el senado de la capital de Rhaetia, Augusta Vindelicorum, P. lulius Pintamus (AE72, 359) y C. lulius Achilleus (NIR 33), y un haruspex, lulius Salutaris (NIR 55). En Noricum constatamos un aedilis de Solva (Seggau, Leibnitz, Austria), lulius Sabinus 1 (CIL III 5345), y un decurio de Celeia, T. Iulius Bellicus (CIL III 15205.3).

Pero los cargos más abundantes entre los varones son, sin duda, los militares, aunque en ningún caso suponen un servicio mayoritario de los Iulii masculinos en el ejército. Encontramos a miembros de la familia tanto en cuerpos auxiliares ${ }^{12}$ como en legiones ${ }^{13}$. El status de las provincias de Rhaetia y Noricum no les permitió tener legiones hasta finales del s.II, cuando pasan de la categoría de procuratorianas a la de pretorianas (WACHER, 1987, 76, 224; MARTIN, $1990,104-106)$, coincidiendo con un aumento

\footnotetext{
11 PFLAUM,I,1960-1961,174, dominus de tres esclavos, Amandus, Maturus y Mercator.

${ }^{12}$ En Rhaetia: IBR 208, lulius; CIL III 5907, Iulius Genialis; CIL III 5918, Iulius Maximus; CIL III 5819, lulius Octavus; AE72, 359, P. lulius Pintamus; NIR 125.1, lulius Rullus; En Noricum: CIL III 15205.3, T. Iulius Bellicus; CIL III 4844, Ti. lulius Buccio; CIL III 4846, Ti. Iulius Capatius; AE54, 100, T. lulius Fronto; CIL III 11554, Ti. Iulius Sextus; CIL III 4847, Ti. lulius Taulus.

${ }^{13}$ En Rhaetia: CIL III 5953, Iulius, T. Iulius Aelianus; CIL. III 5814, lulius Amandus; EE-IV-630, lulius Cattanus; CIL III 5816, lulius Clemens; NIR 51, C. lulius Faventinus; CIL III 14370.2, Iulius lulinus; CIL III 5815, lulius Macrianus; EE-IV. 630, lulius Saturninus; En Noricum: CIL III $5681 \mathrm{~A} / 5$, lulius; CIL III 5655, C. lulius Agricola; CIL III 4842, Iulius Aprilis; CIL III 4843, C. Iulius Bassus, L. Iulius Praesens; CIL III 5631, lulius Exoratus; CIL III 5460, C. Iulius Probus; CIL III 5671 , lulius Apricius.
} 
de los efectivos acantonados tras la crisis de las Guerras Marcomanas.

En Rhaetia este grupo gentilicio ofrece una brillante participación en el ámbito profesional civil, la cual gira en torno a un sector dinámico como es el del comercio. Es un hecho altamente significativo el que sea precisamente la provincia de Rhaetia, la más modesta y con el menor volumen de testimonios epigráficos de las del Alto y Medio Danubio, la que nos brinde el mayor número de negotiatores, incluso sobrepasando a las dos Pannoniae (GALLEGO, $1997,95,341-342)$. Las fuentes epigráficas confirman así el papel estrátegico excepcional jugado por esta pequeña provincia en las rutas de comunicación del Imperio, siendo un nudo de enlace con Italia, Gallia y Germania, así como con las provincias del Danubio y con la Germania libre (WALSER, 1983). Entre los lulii encontramos a lulius Victor 1 (CIL III 5816), un negotiator vestiarius, y lulius Verax (CIL III 5943), que actúa como curam agens de una dedicación monumental de un grupo de negotiatores en Regina Castra (Regensburg, Alemania) (Itin. Ant., 250), y por lo tanto es probable que él a su vez perteneciera a este grupo profesional. Además este encargo que desarrolla revela que lulius Verax gozaba sin duda de cierto prestigio y liderazgo entre sus compañeros de profesión. Finalmente, en el sector de las profesiones liberales los luliiparticipan con un medicus ocularius, C. Iulius Stephanus (NIR 130), quizá un antiguo esclavo griego que ingresó en la familia caesaris ${ }^{14}$.

Por el contrario, los Iulii noricanos no aparecen significativamente vinculados con el mundo del comercio o las profesiones liberales. La única profesión explicitada en nuestra documentación es la de seis centonarii (bomberos), todos ellos pertenecientes al collegium centonariorum de Solva (IRS 149), lo que refleja una notable presencia del grupo gentilicio de los lulii en la ciudad en los inicios del s.lll d.C.. Es posible también que la dedicación votiva de $C$. lulius lustus (AE734, 71) a Savus y Adsalluta, es decir al río Sava y a otro curso fluvial próximo a Celeia, sea reflejo de los intereses profesionales de este individuo en el mundo del transporte y los intercambios (HARDING y JACOBSEN, 1989, 227-232). Sin embargo, pese a esta escasa representación de los cargos municipales o profesionales, sí que se detecta entre los /ulii de Noricum la presencia de un sector con poder económico, probablemente basado en la posesión de propiedades rurales, un grupo acomodado al que pertenecía, por ejemplo, lulia Ingenua 4 (ILI 375), esposa de un te- rrateniente de Sempeter (Yugoslavia), de la familia de los Vindonii (SOLIN y SALOMIES, 1988, 209; HOLDER, III, 1962, 349), importantes propietarios de tierra del sur de Noricum a mediados del s.l d.C.. Este grupo privilegiado manifestaría su influencia a través de la posesión de esclavos y el patronazgo de libertos. De hecho, entre los lulii que son domini y patroni predominan individuos libres, de buen nivel económico, pero a los que no se les conoce desempeño de cargos públicos. Merecen destacarse, por ejemplo, lulius Moderatus ${ }^{15}$, Iulius Priscus ${ }^{16}$, G. Iulius Vogitoutus ${ }^{17}$ o lulia Cassia ${ }^{18}$.

Ya señalamos anteriormente que los individuos de origen servil son un grupo minoritario entre los lulii en estas provincias. En Rhaetia sólo en uno de los libertos menciona explicitamente su status, C.Iulius Libertus, cuyo patrono era C. lulius Silanus (CIL III 5831). En los otros son los cognomina griegos o sus circunstancias familiares los que nos hacen sospechar que pertenezcan a este grupo. Es el caso de la homonimia del haruspex lulius Salutaris (NIR 55) y su esposa lulia lustina, o de lulia Threpte (AE05, 15; SOLIN, 1982, 986, 987), o bien de lulius Marinus (CIL III 5838), que en un epígrafe funerario aparece vinculado a otro individuo que expresa su status de liberto, y del ya citado medicus ocularius C.lulius Stephanus (NIR 130; SOLIN, 1982, 1182, 1368), quizá un liberto imperial.

Entre los /ulii con status de libertos $(18,8$ $\%$ ) de Noricum, la élite la constituyen los libertos imperiales. A este grupo debía pertenecer $C$. Iulius Hermes (CIL III 5036; SOLIN, 1982, 342, 1361), procurator ferrariarum, aunque no explicita su status. Precisamente uno de los principales sectores de la economía de Noricum eran la minería, y el hierro que se extraía allí tenía fama en todo el Imperio (ALFOLDY, 1970, 163177; DUSANIC, 1977, 52-94). También lulia Stratonice (CIL III 4712; SOLIN, 1982, 146) era liberta imperial, ya que su esposo Aquilinus es

\footnotetext{
${ }^{14}$ WEAVER, 1972,82 , donde se señala cómo no es raro que médicos de origen liberto, o incluso libre, aprovechen la coincidencia de su nomen con el del emperador para hacer suponer un servicio a la casa imperial.

${ }^{15} \mathrm{CIL} \mathrm{III} \mathrm{11767,} \mathrm{dominus} \mathrm{de} \mathrm{Speratus,} \mathrm{Peregrina} \mathrm{y} \mathrm{Peregrinus.}$

${ }^{16} \mathrm{CIL}$ III 11497, dominus de Antonia y Cloutius.

${ }^{17}$ CIL III 4908, patronus de Daphinus, Secunda, Aster y Fronto; HOLDER, III, 1962, 435, Destaca el cognomen celta y su condición de indígena romanizado.
}

${ }^{18} \mathrm{AE} 73,392$, patrona de lulius Acceptus y lulia Quinta 2. 
un Caesaris servus, y en este grupo los matrimonios tienden a ser endogámicos (SERRANO, $1988,52-72$ ).

Los libertos privados de los /uliinoricanos no siempre manifiestan abiertamente que éste es su status. Al menos la mitad de ellos no lo hacen, y es su onomástica, especialmente los cognomina griegos (SOLIN, 1971, 159), o sus lazos familiares, los que evidencian su categoría de libertos. Algunos demuestran disfrutar de una posición económica privilegiada, e incluso son a su vez patronos de otros libertos, como lulius Dius (CIL III 5453), que es patrono de tres libertos, Iulius Amianthus, Iulia Amanda y Iulia Quinta 1. Su cognomen griego (SOLIN, 1982, 651) nos hace suponer que el propio Dius era liberto. Es interesante la aparición de las figuras de dos alumni, lulius Auxanon y lulius Eutychianus (SOLIN, 1982, 1263, 807, 1362), dos libertos que quedaron desamparados por su patrono y se colocan bajo la protección de un dominus, Valerius Claudius Quintus (CIL III 4855; CRESPO, 1992, 225-239). Los que manifiestan directamente su categoría de libertos, emplean la fórmula tradicional del praenomen del patrono en genitivo, aunque en ocasiones se cita el cognomen del patrono en genitivo. Esta práctica no es mayoritaria, pero tampoco es extraña, habiéndose constatado ya en Italia ${ }^{19}$. Entre los lulii de Noricum en unos casos el patrono así citado es a su vez un liberto (CIL III 5453), pero tampoco podemos excluir que otros sean hombres libres (CIL III 4926, 5644, 4845). La mención del cognomen del patrono podría tener una finalidad identificativa entre varios miembros de una familia, todos patronos de libertos. Además, también existe la práctica de citar la filiación de un individuo mediante el cognomen del padre, en vez de mediante su praenomen, no sólo entre los lulii de Noricum (CIL III 4838, 15205.3, 11554), sino en la propia Italia (por ejemplo, CIL V 3957, 3958).

Finalmente, nos quedaría por abordar el análisis del comportamiento religioso-votivo de la familia en ambas provincias que nos revela sus preferencias cultuales. Son los varones de la gens /ulia, tanto en Rhaetia como en Noricum, los que toman la iniciativa en el campo votivo, y las dedicaciones femeninas son minoritarias. Pero tanto entre los hombres como entre las mujeres, la mayoría de los dedicantes son personas libres. Predominan las dedicaciones a divinidades romanas. En Rhaetia las dedicaciones más frecuentes son a Victoria y a luppiter Optimus Maximus, precisamente dos de las divinidades representantes del Estado romano y del culto oficial, lo que vincula a los lulii $\sin$ duda alguna con las estructuras oficiales del Imperio. lulius Iulinus y lulius? Farianus? (CIL III 5944) pudieron ser los quaestores de una asociación de cultores que dedican a Victoria por la salud de Severo Alejandro en Regina Castra, aunque el cargo que desempeñan podría referirse también a la organización municipal de esta ciudad. En este contexto de oficialidad, aunque referida a una divinidad oriental, hay que situar también la dedicación del legatus Augusti $G$. lulius Avitus Alexianus (AE62, 229) a Deus Patrius Solielagabalus, con la que el gobernador de Rhaetia apoya el nuevo culto oficial e imperial instaurado por Heliogábalo. Los epítetos Deus Patrius colocan al dios en la categoría de señor supremo protector del Estado romano. Otras divinidades romanas honradas entre los lulii réticos son Mercurius y Vulcanus. Los dedicantes a Mercurius, el dios de los negocios, el comercio y los viajes, son los negotiatores de Regina Castra ${ }^{20}$, y lulius Gallicus (CIL III 5877), que pone un signum del dios. Finalmente, el único cargo religioso que conocemos entre los lulii réticos es desempeñado por un hombre, el haruspex lulius Salutaris (NIR 55), que dedica a luppiter Optimus Maximus, aunque su cargo parece vincularse más bien al cercano mithraeum de Faimingen (Alemania) (IBR 214). En las dedicaciones femeninas constatamos otras divinidades romanas, como la pareja DisProserpina (CIL III 11923), o las FontanaeNymphae (AE05, 15), más ligadas con el mundo ctónico y las fuerzas fecundas de la naturaleza, mientras que las prácticas votivo-religiosas de los varones les vinculan a divinidades asociadas con el ámbito socioeconómico y profesional, y con la vida pública y oficial.

Los cultos romanos también están bien representados entre los Iulii de Noricum especialmente al dios supremo, protector del Estado, luppiter Optimus Maximus, pero también a Fortuna Augusta y Mercurius Augustus. Estas dedicaciones a Júpiter Capitolino vinculan a los dedicantes con las estructuras ideológicas del Imperio, como el miles legionis $X$ Gemina $C$. lulius Probus (CIL III 5460), o lulius Cogitatus y lulius Primillus, que pertenecen a una asociación de cultores de Solva, cuyos miembros ofre-

\footnotetext{
${ }^{19}$ por ejemplo, CIL V 436, 515, 804, 915, 1101, 1135, 2882, $3300,3410,3901$; ZAJAC, 1990-1992, 350-359.

${ }^{20} \mathrm{CIL}$ III 5943, restauran un aedes cum signum de Mercurius Censualis, en honor, además, de la Domus Divina, acto del que es curam agens lulius Verax.
} 
cen una dedicación monumental. Este carácter oficial se refleja igualmente en las consagraciones a Fortuna y Mercurius, contenidas en placas monumentales de mármol puestas por collegia de los que los lulii forman parte, concretamente el collegium Manliensium de Virunum (CIL III 4778, 4785, a Fortuna Augusta), - la asociación de cultores de Celeia (CIL III 5196, a Mercurius Augustus). Otras divinidades romanas que reciben culto son Hercules (AE78, 605) y Dis o Plutón, divinidad ctónica honrada por una mujer, lulia Gemellina (AE50, 98). Un culto de características peculiares es el que reciben los cursos fluviales, cuyos dedicantes se vinculan probablemente a actividades de transporte, como C. lulius lustus, que consagra a Savus et Atsalluta (AE34, 71). Los cultos orientales son minoritarios entre los lulii, aunque se atestiguan algunos de los más exitosos del Imperio: Mithra e Isis. En Noricum, Mithra encuentra predicamento entre sujetos militares de la familia (CIL III 11548; AE56, 82); igualmente encontramos un número significativo de miembros de la gens en el album de los miembros del mitreo de Virunum, (AE94, 1334, 1335), entre los que destaca por su cargo el pater lulius Secundinus, y que testimonian los lazos estrechos entre el culto mitraico y la ideología oficial, ya que ambas dedicaciones se consagran por la salud de emperadores reinantes (Commodo, Septimio Severo y Caracalla). Isis Augusta (AE56, 44) en honrada en Noricum por C. Iulius Onesimus, posiblemente liberto de procedencia oriental, y su hija lulia Ingenua 3. En Rhaetia ya nos hemos referido a la dedicación votiva al Deus Patrius Solielagabalus.

Este predomonio de las divinidades romanas, a veces asociadas a fenómenos de asociacionismo religioso, refleja un significativo grado de romanización y asimilación de las estructuras ideológicas romanas entre los lulii de nuestras provincias. Sin embargo, también constatamos claramente en la familia la pervivencia de cultos y divinidades de raíz prerromana indígena, aunque sea de forma puntual. En Rhaetia estos cultos afloran en ocasiones en fenómenos de interpretatio (MARTIN, 1991, 228-230), como evidencia la dedicación de Iulia Matrona a Apollo Grannus, Diana Sacta y Sirona (AE92, 1304; HOLDER, I, 1961, 1286, 2037). Otros conservan su nombre indígena: las diosas Suleviae ${ }^{21}$ son honradas por una mujer, Iulia Paterna 2 (CIL III 5900) y Gontia por un varón, el centurio de la legio / Italica C.lulius Faventinus (NIR 51). En Noricum la pervivencia de antiguos cultos indígenas se rastrea tanto en dedicaciones masculinas como femeninas. En ocasiones presentan una forma latina, como los luvantes (CIL III 5118), pero no siempre: Belestis Augusta (CIL III 4773; HOLDER, I, 1961, 374), C/bus? Augustus (CIL III 4721; HOLDER, I, 1961, 651) o Vocretanus Augustus (AE59, 150A).

En el ámbito funerario los /ulii emplean la consagración a los Dii Manes o la Perpetua Securitas, a menudo asociadas en la epigrafía funeraria de Rhaetia. Su aparición sin embargo no es frecuentemente en la familia, especialmente en Noricum, lo que evidencia un retraso en la penetración de esta fórmula en esta provincia frente a otras del Imperio, a lo que hay que unir el hecho de que los lulii se extiendan fundamentalmente durante el s.I y II d.C., mientras que estas fórmulas funeraria proliferan en el s.lll d.C..

Los lulii se concentran en Rhaetia en torno a dos de los principales núcleos provinciales, la capital, Augusta Vindelicorum, y Regina Castra, en el limes danubiano, siendo poco significativa la presencia de la familia en otras zonas, aunque cabe destacar que parte de ellos se encuentran de forma difusa a lo largo del limes. También en Noricum se aprecia la preferencia de la familia por los asentamientos urbanos, o su entorno más inmediato, entre los que cobra protagonismo la capital, Virunum, y en segundo lugar Solva y Celeia. Apenas una cuarta parte de los /ulii noricanos se sitúan en áreas rurales, alejadas de los núcleos urbanos de primer orden, bien en la zona de Carintia (Kärnten) o Estiria (Steiermark). En definitiva, mientras en Rhaetia la gens lulia tiende a situarse en la franja del limes, en Noricum se centra en la zona sur provincial, donde se concentran los principales centros urbanos y administrativos y las zonas de explotación comercial y minera.

Finalmente, la familia presenta variaciones significativas en su distribución cronológica en ambas provincias. Aproximadamente una cuarta parte de los lulii de Rhaetia se concentran en el s.l y primera mitad del s.II d.C., frente a las casi tres cuartas partes que se extienden desde mediados del s.ll y durante el s.III d.C., registrando una presencia meramente testimonial de la familia en el s. IV d.C. En Noricum la distribución de los luliien el tiempo manifiesta que casi las tres cuartas partes de ellos se concentran en los dos primeros siglos del Imperio. Hay una sólida, aunque minoritaria, implantación de la familia ya en el s.l, la cual se extien-

\footnotetext{
${ }^{21}$ HOLDER, III, 1962, 1663, divinidades de tipo curativo.
} 
de con vigor en el s.ll, para ir reduciendo su presencia en el s.III y IV, comportamiento que puede ser simplemente paralelo a un descenso general de los testimonios epigráficos a partir del s.III, hecho en el que pudo tener su incidencia, ya en las últimas décadas del s.II, las Guerras Marcomanas (166-180 d.C.).

En conclusión, el primer momento de la implantación del gentilicio en Rhaetia y Noricum coincide con la primera etapa de la romanización impulsada por Augusto y Tiberio, quienes probablemente concedieron la ciudadanía a título individual y de forma restringida a miembros de la aristocracia indígena (MARTIN, 1991, 33-35), y así lo demuestra, entre los varones que usan praenomen, el predominio de la asociación $C$. lulius. También hay que considerar las aportaciones de emigrantes de origen itálico y occidental, como nuestro análisis ha puesto de manifiesto. Sin embargo, la gran expansión de los lulii se produce en el s.II, incluso durante su etapa final y las primeras décadas del s.lll en Rhaetia, cuando el proceso romanizador es más maduro y los testimonios epigráficos más abundantes, de manera que la familia se convierte asi en la más importante desde el punto de vista cuantitativo y cualitativo de nuestras provincias. Este proceso se ve alentado por el acceso a las magistraturas municipales de algunos de sus miembros, mientras que otros, aunque alejados de cargos públicos municipales, manifiestan una posición económica desahogada, como hemos atestiguado en Noricum, y extienden su influencia a través de sus esclavos y libertos, y probablemente otras relaciones de clientela. Otros alcanzan la ciudadanía a través del servicio militar en los cuerpos auxiliares.

Además, no podemos descartar otras influencias en la expansión del nomen en un momento más avanzado, en el período Severo, antes de la Constitutio Antoniniana (212 d.C.), e incluso después de ella, como ocurre en Rhaetia. El gentilicio lulius/a se halla muy presente en esta dinastía a través de la onomástica de las influyentes emperatrices severas, especialmente lulia Domna, esposa de Septimio Severo y ma- dre de Caracalla, que aparece asociada a sus hijos Caracalla y Geta en varias dedicaciones del culto imperial en Rhaetia (CIL III 5935, 11951, del reinado de Caracalla). Sabemos además que otros miembros de esta familia ocuparon puestos de la mayor relevancia en la provincia, como el legatus Augusti G. Iulius Avitus Alexianus, que comenzó su carrera con Septimio Severo, siendo gobernador de Rhaetia bajo Heliogábalo (AE21, 64; AE62, 229; $\mathrm{PIR}^{2}$ I 192). Finalmente, la persistencia testimonial de este grupo gentilicio tanto en Rhaetia como en Noricum hasta época tardía, s.IV d.C., cuando el uso del gentilicio cae en desuso y la documentación epigráfica provincial es tan escasa, confirma una vez más a los lulii como la principal familia gentilicia de estas provincias, y refleja el vigor y arraigo del nomen lulius en las mismas.

\section{ABREVIATURAS DE LOS CUADROS DE DATOS EPIGRAFICOS}

Campo Prae: Praenomen.

Campo Etn (Tipo de cognomen): $R=$ Romano, I= Indígena, $\mathrm{G}=$ Griego.

Campo Sta (Status): $D=$ Dominus (de esclavos), $E=$ Equestre, I= Ingenuus, L= Liberto, LA= Liberto de Augusto, $\mathrm{LP}=$ Liberto y patrono, $\mathrm{P}=\mathrm{Patrono}$ (de libertos) $\mathrm{S}=\mathrm{Sier}$ vo, $\mathrm{SN}=$ Senador.

Campo Lugarhalla (Lugar de Hallazgo): $\mathrm{AL}=$ Alemania, $A L T=$ Althofen, $A U=$ Austria,$A \cup G=$ Augsburg, $B A=B a-$ yern, $B R=B r u c k, C E=$ Celje, $D R=D r a v o g r a d, E I=E i c h s t a ̈ d t$, $E N=E n n s, F E L=$ Feldkirchen, $F R=F$ riul, $F R S=$ Friesach, $\mathrm{GL}=$ Gleisdorf, $\mathrm{GR}=\mathrm{Graz}, \mathrm{GU}=\mathrm{Günzb}$ urg, $\mathrm{HA}=$ Hartberg, $\mathrm{HEI}=$ Heidenheim, IN= Ingolstadt, IT = Italia, $K A=$ Kärnten, $K L A=$ Klagenfurt, $K E=$ Kelheim, $K R=K r e m s, L A F=$ Laufen, $L E=$ Leibnitz, LIE= Liezen, LI= Linz, MA= Mauthen, MAR= Maribor, $M I=$ Mindelheim, NEM = Neumarkt, NEU= Neuburg, $\mathrm{NI}=$ NiederÖsterreich, $\mathrm{NO}=$ Noricum, $\mathrm{OBO}=$ OberÖsterreich, OS= Osttirol, REG= Regensburg, $\mathrm{RH}=$ Rhaetia, $R O=$ Rosenheim, $S A=$ Salzburg, $S A L=$ Salzburg, $\mathrm{SB}=$ Slov. Bistrica, SL= Slovenija, ST= Steiermark, STP= St. Pölten, STR $=$ Straubing, $T I=$ Tirol, $T I T=$ Titovo-Velenje, $\mathrm{TR}=$ Traunstein, $\mathrm{TRB}=$ Trbovlje, $\mathrm{TRO}=$ Trostberg, $\mathrm{TU}=$ Tulln, VIL $=$ Villach, $V O=$ Völkermarkt, $V O C=$ Vöcklabruck, $W E L=$ Wels, $W O=$ Wolfsberg, $Y U=$ Yugoslavia .

Campo Crono (Cronología)

Campo Biblio (Bibliografía): cfr. nota 4. 
PRAE NOMEN COGNOMEN

IULIA1

IULIA1

IULIA ALPINA

IULIA ATTILIA

IULIA IANUARIA

IULIA IUSTINA

IULIA LEONTIA

IULIA MATRONA

IULIA MATTA?

IULIA PATERNA

IULIA PATERNA2

IULIA SECUNDINA

$\begin{array}{ll}\text { IULIA } & \text { TERTIOLA } \\ \text { IULIA } & \text { THREPTE }\end{array}$

IULIA URSA 1

IULIA UASA2

IULIA VENULANI

IULIA VERA

IULIA VICTORINA

IULLA VICTORINA

IULIA? IULIANA

IULIA? QUIETA

IULIUS 1

IULIUS 2

IULIUS 3

ULIUS 5

IULIUS 6

c. IULIUS7

c. IULIUS 2

C. IULIUS

c. IULIUS ACHILleUS

IULIUS AELIANUS

IULIUS ALPINUS
IULIUS AMANDUS

IULIUS ANTONINUS
IULIUS AUGUSTANUS

G. IULIUS AVITUS ALEXIANUS

IULIUS CATTANUS

IULIUS CATTANUS
IULIUS CLEMENS

IULIUS CLEMENS
IULIUS COGITATUS?

IULIUS CONCESIANUS?

IULIUS CRESCENS?

IULIUS DUBITATUS

c. IULIUS FAVENTINUS

IULIUS FLORIANUS

IULIUS FLORINUS

IULIUS GALIICUS

IULIUS GENIALIS

c. IULIUS IMPETRATUS

IULIUS IULIANUS

IULIUS IULIANUS

IULIUS IULINUS

IULIUS IUSTUS

IULIUS LEONTIUS

c. IULIUS LIBERTUS

IULIUS LUCILIANUS

IULIUS MACRIANUS

IULIUS MARCIANUS

IULIUS MARINUS

IULIUS MASUETUS

IULIUS MAXIMUS

IULIUS MODESTINUS

IULIUS MODESTUS

IULIUS NOVELLUS

IULIUS OCTAVUS

P. IULIUS PINTAMUS

c. IULIUS POTITIT

IULIUS PRISCINUS

IULIUS QUARTUS

IULIUS QUIETUS

IULUS QUINTINUS

IULIUS QUINTUS?
IULIUS RESPECTUS

IULIUS RESTITUTUS

IULIUS RULLUS

IULIUS SALUTARIS

IULUS SATURNIINUS

ULLUS SECUNDINUS

SEVERUS

IULIUS STATUTIANUS

IULIUS STEPHANUS

IULIUS TETRIGUS

UULUS VALENTIANUS

IULIUS VASTUS?

IULIUS VERAX

IULIUS VERUS

IULIUS VIATOR

IULIUS VICTOR 1

ULIUS VCTOR 2

IULIUS VICTORIAN

IULIUS-A

IULIUS-A

IULIUS-A

IULIUS? DEOTITANUS

IULIUS? FARIANUS?

IULIUS? IULIANUS

Tabla 1. Iuliien Rhaetia.
ETN STA DIVINIDAD

DII MANES

L? IUPPITER OPTIM. MAX.

APOLLO,DIANA,SIRONA

DIIMANES

DIIMANES

SULEVIAE

L? FONTANAE-NYMPHAE

DII MANES-SECURITAS

DII MANES

DII MANES-SECURITAS

ICTORIA

SN SOLELAGABALUS

DII MANES

GONTIA

VICTORIA

MERCURIUS

IUPPITER OPTIM. MAX.

VICTORIA

(1)

VULCANUS

IUPPITER OPTIM. MAX

VICTORIA
VICTORIA

DII MANES-SECURITAS

MERCURIUS

VICTORIA

UPPITER OPTIM. MAX

? IUPPITER OPTIM. MAX

DIIMANES

DII MANES

VICTORIA

IUPPITER OPTIM. MAX.

VICTORIA

VICTORIA

P?

VICTORIA
VICTORIA
LUGARHALLA

RH-AL-BA-EI-Ptünz

RH-AL-BA-Augsburg

RH-AL-BA-Augsburg

RH-AL-BA-Heidenheim

RH-AL-BA-GU-Gundremmingen

RH-AL-BA-Augsburg

RH-AL-BA-Augsburg

RH-AL-BA-Regensburg

RH-AL-BA-Augsburg

RH-AL-BA-El-Nassenfels

RH-AL-BA-Augsburg

RH-AL-BA-Augsburg

RH-AU-TI-Matzen

RH-AL-BA-Regensbuzg

RH-AL-BA-Regensburg

RH-AL-BA-Regensburg

RH-AL-BA-Augsburg

RH-AL-BA-Regensburg

RH-AL-BA-Regensburg

RH-AL-BA-IN-Pföring

RH-AL-BA-KE-Eining

RH-AL-BA-Regensburg

RH-AL-BA-Regensbur

RH-AL-BA-Augsburg

RH-AL-BA-Regensburg

RH-AL-BA-Regensburg

RH-AL-BA-REG-Alkofen

RH-AL-BA-El-Pfünz

RH-AL-BA-AUG-Leutstetten

RH-AL-BA-Augsburg

AH-AL-BA Regensbur

RH-AL-BA-Regens

RH-AL-BA-Augshurg

RH-AL-BA-Augsburg

RH-AL-BA-Augsburg

RH-AL-BA-REG-Wienting

AH-AL-BA-Augsburg

RH-AL-BA-Augsburg

RH-AL-BA-Regensburg

RH-AL-BA-Augsburg

BH-AL-BA-EI-Pfünz

RH-AL-BA-KE-Eining

RH-AL-BA-Günzburg

RH-AL-BA-REG-Wienting

RH-AL-BA-REG-Wienting

RH-AL-BA-Lauingen

RHH-AL-BA-IN-Kösching

RH-AL-BA-El-Nassenfels

RH-AL-BA-REG-Wienting

RH-AL-BA-REG-Wienting

RH-AL-BA-El-Kipfenberg

RH-AL-BA-AUGSbur

RH-AL-BA-Augsburg

RH-AL-BA-Regensburg

RH-AL-BA-Augssburg

RH-AL-BA-Augsburg

RH-AL-BA-Augsburg

RH-AL-BA-EI-Pfühz

RH-AL-BA-REG-Wienting

RH-AL-BA-AEG-Wienting

RH-AL-BA-Weissenbur

RH-AL-BA-Augsburg

RH-AL-BA-AUG-Leutstetten

RH-AL-BA-Regensburg

RH-AL-BA-GUN-Gnotzheim

RH-AL-BA-Weissenburg

RH-AL-BA-REG-Wienting

Rh-AL-BA-Regensburg

RH

RH-AL-BA-Regensbu

BH-AL-BA-El-Eining

RH-AL-BA-Straubing

RH-AL-BA-GU-Gundremmingen

RH-AL-BA-Regensburg

RHA-BA-Augsburg

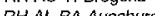

BH-AL-BA-Augsburg

RH-AL-BA-Straubing

RH-AL-BA-PEG-Wienting

RH-AL-BA-ML-Türkheim

RH-AU-TI-Bregenz

RH-AL-BA-Regensburg

RH-AL-BA-Aug-ESteing

RH-AL-BA-Augsburg

RH-AL-BA-REG-Wienting

RH-AL-BA-REG-Wienting

$\mathrm{RH}$-AL-BA-Regensburg

RH-AU-TI-Matzen

RH-Al.BA-Augsburg

RH-AL-BA-GU-Gundremmingen

RH-AL-BA-Regensburg

RH-AL-BA-REG-Wienting

CRONO BIBLIO

FII-III CIL-III-5813

FIIIII CIL-III-5815

MIII-III NIR-32
III CIL-III-11923

FIIIIII ClL-III-5956

FIII-III NIR-55

IV AE78-577

CIL-1II-5836

FII-III CLL-III-5955

FI1-III CIL-III-5814

AE80-661

FII-III CIL-III-5955

FII-CIII CIL-1II-5950

MII-MIII CIL-III-5965

NIIIFII AE:80-661

$\begin{array}{ll}\text { FIJ-III } & \text { CIL-III-5955 } \\ \text { FII-III } & \text { CIL-14370.15 }\end{array}$

II IBR-264

H-CIII CIL-III-15211.1

FII-III CIL.111-5953

I-III CLL-III-11974

FI-MH ClL-III- 1197

FI-MII IBR-208

I-III CIL-14115.49

III CIL-III-5934

CIL-12010

CIL-12010.18

CIL-1II-11975

NII-3H

CIL- $111-5953$

CIL-III-5814

NIR-33

CIL-III-594

AE62-229

AE62-229
EE-IV-630

CIL-III-5816

H-CIII CIL-III-5848

I-iil CIL-14116.14

II CIL-III-5936

CIII CIL-III-5944

III CIL-III-5877

FI-CII CIL-III-5907

MII-III CIL-III-5894

CIII CIL-III-5944

CIII CIL-III-5944

II-CIII ClL-III-5882

IV AE78-577

FII-II! CIL-III-6831

FII-III CIL-III-5815

MII-III CIL-III-5799

FI-II ClL-|H-5838

Mil-CIII CIL-III-15211.2

CIII CIL-III-5918

CIII CIL-III-5944

H-III CIL-14116.15

II AE72-359

MII-MIII CLL-III-5965

I-II CIL-III-5926

CHI CIL-III-5944

MII-CIII CLL-III-15211.

AII-FII CLL-III-11967

-MII CIL-14115.48

CIL-12014.31 
PRAE NOMEN COGNOMEN

$\begin{array}{ll}\text { IULIA? } & \text { IULIANA } \\ \text { IULIUS } & \text { IULIANUS 1 } \\ \text { IULIUS } & \text { IULIANUS ? } \\ \text { IULIUS } & \text { IULINUS } \\ \text { IULIUS? } & \text { IULIANUS } \\ & \text { IULLANUS 1 } \\ & \text { IULIANUS 2 } \\ & \text { IULIANUS 3 } \\ & \text { IULIANUS? } \\ & \text { IULLINUS } \\ & \text { IULINUS }\end{array}$

ETN STA DIVINIDAD

$R$
$R$
$R$
$R$
$R$
$R$
$R$
$R$

VICTORIA
VICTORIA

VICTORIA

IUPPITER OPTIM. MAX

$\begin{array}{lll}R & \text { I } & \text { VICTORIA } \\ R & & \text { VICTORIA } \\ R & \text { I } & \\ R & & \text { VICTORIA } \\ R & 1 & \\ R & & \text { IUPPITEROPTIM. MAX } \\ \text { R } & & \\ \text { R } & & \\ \text { R } & & \\ \text { R } & & \end{array}$

Tabla 2. Cognomina derivados del nomen lulius en Rhaetia.
LUGARHALLA

PH-AL-BA-KE-Eining

AH-AL-BA-KEG-Weinting

RH-AL-BA-REG-Wienting

RH-AL-BA-EI-Kiplenberg

RH-AL-BA-REG-Wienting

PH-AL-BA-STR-Pfelling

PH-AL-BA-Regensburg

RHA.BA-TH

RH-AL-BA-Gúnzburg
CRONO BIBLIO

II CIL-III-5936

CIII CII CIL-III-5944

CIL-III-14370.2

CIL-1II-5944

CII-CIII CIL-III-15211 CIL-6010.106

CIL-14115.47

CIL-12014.310

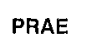

COGNOMEN

ULIA 1

IULIA

IULIA 3

IULIA 4

IULIA 5

IULIA 6
IULIA 7

IULIA 7
IULIA 8

IULIA 9

IULIA 10

IULIA 12

IULIA 13

IULIA 14

IULIA 15

IULIA

IUUIA

IULIA

IULIA

IULIA

IULIA
IULIA
IULIA

IULIA
IULLA
IULIA
IULIA

IULIA

IULIA

IULIA

UULIA

ULIA

IULIA

IULIA

IULIA

IULIA

JULIA

IULIA

ULIA

ULIA

ACCEPTA

CENSORINA

CONCORDIA

EMERITA

EUTYCHIA

EVODIA

EXORATA
FESTA

FIRMINA

GUSGA

HILARA

HONORATA 1

HONORATA

IANUARIA

NGENUA 2

NGENUA

NGENUA

ULIANA

IUNIANA

UTU

LITUGENA

MAXIMINA

MELISSA

MYRINA

NOVELLLA

PRIMITIVA

PROCILLA

PUSILLA

QUINCTILLA

QUINTA 1

RESPECTA

SABINA 1

SABINA 2

SECUNDA 1

SECUNDINA 1

SECUNDINA.

SECUNDINA 3

SECUNDINA

SEROTINA

SEVERIO-SIC

SISIA

STRATONICE

SUCCESSA 1

SUCCESSA 2

TERTULLA

VALENTINA

VEGETA

VENUSTA

VERA

VERECUNDA

VICTORINA

CASSIA

IANUARIA?

INGENUA

INGENUA

ROMULA

ETN STA DIVINIDAD

LUGARHALLA

NO-AURA-KLA-Töltschach

NO-AU-KA-KLA-Obermühlbach

NO-AU-KA-WO-St.Aegidius?

NO-AU-ST-LE-Seggau

NO-AU-ST-Frledberg

NO-AU-ST-LIE-Wörschach

NO-AU-KA.KLLA-S.Georgen am Längsee

NO-AU-KA-ALT-Eberndort

NO-YU-SL-CE-Tomaz

NO-AU-NI-TU-Zwentendor

NO-AU-ST-HA-Löffelbach

NO-AU-ST-HA-Löffelbach

NO-AU-KA-KLA-St.Georgen am Längse

NO-AU-KA-KLA-St.Georgen am Längse

NO-AU-KA-KLA-Breitenstein

NO-AU-KA-KLA-Magdalensberg

NO-AU-ST-GR-Waldstein

NO-YU-SL-TRB-Trojane

NO-AU-KA-KLA-Viktring

NO-AU-KA-WO-S.Paul im Lavantta

NO-YU-SL-TI-S.Veit??

NO-AU-KA-KLA-S.Leonhard am Loibl

NO-AL-BA-TR-Frauenchiemsee

NO-YU-SL-Celje

NO-AU-KA-KLA-Feistrit2

NO-AU-SA-Salzburg

NO-AU-KA-VIL-Saifnitz

NO-AU-KA-KLA-Pöllin

NO-AU-OBO-Wels

NO-AU-ST-LE-Seggau
NO-YU-SL-Celje

NO-YU-SL-Celje

NO-AU-KA-KL_A-Grossbuc

DIS SMERTRIUS

IUPPITER OPTIM. MAX.

IUPPITER OPTIM MAX.

DII MANES

DSISAUGUSTA

C/BUS? AUGUSTUS

ᄂ

L

L?

L

$P$

L?

L?

DII MANES

LA DIIMANES

L?

DII MANES

DII MANES

NO-AU-SA-SAL-Nonnber

NO-AU-ST-GR-Reun

NO-AU-KA-WO-St.Michae

NO-AU-KA-FRS-Hüttenberg

NO-AU-KA-FRS-Hütten
NO-AU-SA-Salzburg

NO-AU-KA-KLA-Töltschach

NO-YU-SL.CE-Sempeter

NO-AU-OS-LI-Görtschach

NO-AU-SA-SAL-Nonnberg

NO-AU-ST-Leibnitz

NO-AU-ST-GL-S.Johann

NO-AU-KA-KLA-Solesson

NO-YU-SL-Celje

NO-AU-KA-KLA-Untermühlbach

NO-AU-ST-LE-Grottenhofen

NO-AU-KA-KLA-Zollfeld

NO-AU-SA-SAL-Nonnberg

NO-AU-KA-KLA-Magdalensberg

NO-AU-ST-GR-Waldstein

NO-AU-KA-KLA-Breitenstein

NO-AU-ST-GL-Hainersdor

NO-AU-KA-KLA-Zollfeld

NO-AU-KA-WO-St.Michael

NO-AU-SA-Salzburg

NO-AU-ST-NEM-Neudeck

NO-AU-ST-LE-Seggau

NO-AU-ST-LE-Landscha

NO-AU-OBO-VOC-Mondsee

NO-AU-KA-KLA-Timenitz

NO-AU-OBO-EN-LorCh

NO-AU-KA-KLA-Viktring

NO-IT-FF-Tarvisio

NO-YU-SL-Celje

NO-AU-ST-LE-Seggau

NO-AU-ST-St.Lambrecht

NO-AU-KA-KLA-Mariasaal

NO-AU-KA-KLA-Tanzenber

NO-AU-KA-KLA-Pölling

NO-IT-FR-Fontebba

NO-YU-SL-Celje

NO-AU-ST-LE-Seggau

NO-AL-BA-RO-Eggstătt

NO-AU-KA-KLA-Mariasaal
NO-AU-KA-KLA-Breitenstein

NO-AU-ST-LE-Kaindorf

NO-AU-ST-GR-Waldstein

NO-AU-ST-HA-Lôtrelbach

NO-AU-KA-KLA-St.Veit

gen am Längse

CRONO BIBLIO

CIL-III-4907

MII-III CIL-III-11668

CIL. || $\mid-5378$

ClL. $11-5520$

-CII ClL-1II-5644

FI-II CIL-ill-4838

CIL-III-11554
CIL-HII-11637

AE89-59

AE $77-597$

IRS-92

IRS-92

CIL-1II-4844

CIL-111- 4844
AE73-392

CIL-III-4924A

CIL-III-5453

CIL-III-1167

CIL-III-5586

FI-CII CIL-III-4933

FII-III C|L-III-5106

CIL-IIII-5106
CIL-III- 4773

AE66-276

I-II CIL-III-523

$\begin{array}{ll}\text { FI+II } & C \mid L-I I I-4838 \\ \mid+11 & C \mid L-I I I-5543\end{array}$

CIL-IIII-5543
CIL-III-4717

CIL-III-11580

CIL-III-5631

CIL-111-5353

CIL-III-5268

CIL-III-4998

MI-II AE AL-98

I-MII CIL-III-5544

MII-MIII CIL-III-5582

CIL-III-5090

MII-MIII CIL-III-5548

FI-CII AE56-44

ILI-375

I-CII CIL-III-5545

FI-II CIL CL-III-5501

FI-MIFI CIL-III-15205C

CII-1II-5233

CIIL-III-11560

CIL-III- 4845

AE89-595

CIL-III-4923 


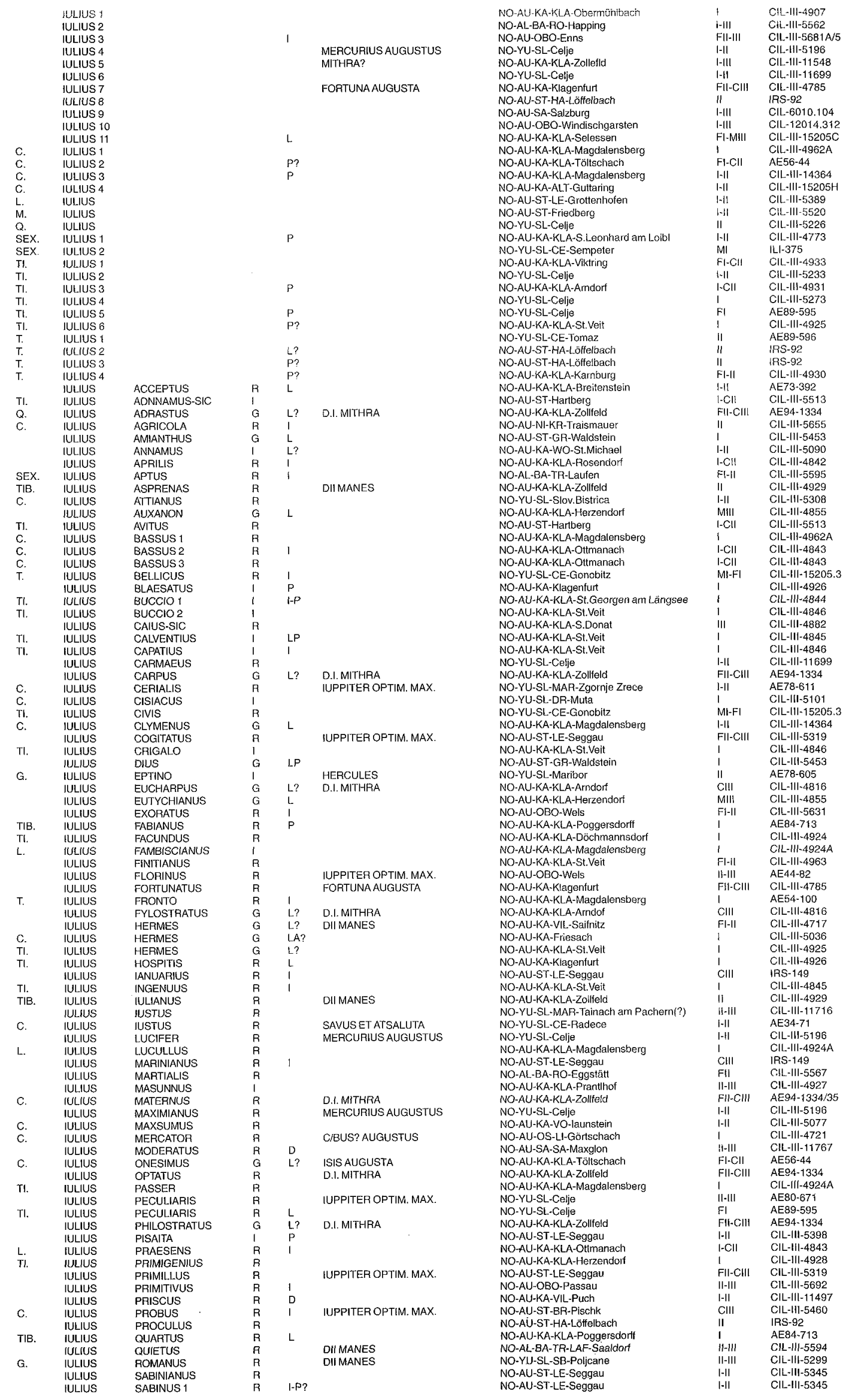




\begin{tabular}{|c|c|c|c|c|c|c|c|c|}
\hline & IULIUS & SABINUS 2 & $\mathrm{R}$ & & & NO-AL-BA-TR-Frauenchiemsee & $\|$ & AE66-276 \\
\hline & IULIUS & SATURNINUS & $\mathrm{B}$ & i & & NO-AU-ST-LE-Seggau & CIII & IRS-149 \\
\hline \multirow[t]{6}{*}{ T. } & IULIUS & SATURNINUS & $\mathrm{R}$ & E.D & & NO-AU-KA-MA-Reisach & MII & CIL-III-4720 \\
\hline & IULIUS & SECUNDINUS 1 & $\mathrm{R}$ & & SECURITAS PERPETUA & NO-AU-ST-LE-Landscha & FIIIIII & CIL-III-5362 \\
\hline & IULIUS & SECUNDINUS 2 & $\mathbf{R}$ & & & NO-AU-OBO-VOC-Mondsee & $\mathrm{Fl}-\mathrm{-I}$ & CIL-III-5626 \\
\hline & IULIUS & SECUNDINUS 3 & $\mathrm{R}$ & & FORTUNA AUGUSTA & NO-AU-KA-KLA-Töltschach & $\mathrm{Fl}[-\mathrm{C} H]$ & CIL-III- 4778 \\
\hline & IULIUS & SECUNDINUS 4 & $\mathrm{~B}$ & 1 & & NO-AU-ST-LE-Seggau & Cill & IRS-149 \\
\hline & IULIUS & SECUNDINUS 5 & $\mathrm{R}$ & $i$ & D.I. MITHRA & NO-AU-KA-KLA-Zollfeld & FII-CM & AE94-1334 \\
\hline \multirow[t]{2}{*}{ c. } & IULIUS & SECUNDINUS & $\mathrm{R}$ & & D.I. MITHRA & NO-AU-KA-KLA-Zollfeld & FII-CHII & AE94-1334 \\
\hline & IULIUS & SECUNDUS & $\mathrm{R}$ & & & NO-AU-OBO-VOC-Mondsee & FI-II & CIL-HII-5626 \\
\hline c. & IULIUS & SECUNDUS & $\mathrm{R}$ & & C/ABUS?AUGUSTUS & NO-AU-OS-LI-Görtschach & 1 & ClL-III-4721 \\
\hline TIB. & IULIUS & SENECIO & $\mathrm{R}$ & & & NO-AU-KA-KLA-Zollfeld & II & CIL-III-4929 \\
\hline $\mathrm{TI}$. & IULIUS & SEVERUS & $\mathrm{R}$ & & & NO-YU-SL-Celje & i & CIL-III-5273 \\
\hline TI. & IULIUS & SEXTUS & $\mathrm{R}$ & I-P & & NO-AU-KA-KLA-S.Georgen am Längsee & i & CIL-III-11554 \\
\hline T. & IULIUS & sossius & 1 & L? & & NO-AU-KA-KLA-Karnburg & FI-II & Cll-1II-4930 \\
\hline \multirow[t]{2}{*}{ TI. } & IULIUS & SUCCESSUS & $\mathrm{R}$ & $\mathrm{L}$ & & NO-AU-KA-KLA-Aindorf & $1-\mathrm{CAl}$ & CIL-NII-4931 \\
\hline & IULIUS & TACITIANUS & $\mathrm{R}$ & 1 & & NO-AU-ST-LE-Seggau & Clil & IRS-149 \\
\hline \multirow[t]{3}{*}{ TI. } & IULIUS & $\begin{array}{l}\text { TALLUS } \\
\text { TAUS }\end{array}$ & $i$ & $i$ & & NO-AU-KA-KLA-Magdalensberg & 1 & CIL-11I-4847 \\
\hline & IULIUS & TERTIANUS & $\mathrm{A}$ & & & NO-AU-KA-KL-A-Mariasaal & $\mathrm{FI}-\mathrm{II}$ & CIL-1II-4971 \\
\hline & IULIUS & TERTIUS & A & & VOCRETANUS? AUGUSTUS & NO-AU-KA-FEL-Ossiach & $|-| \mid$ & AE59-150A \\
\hline \multirow[t]{3}{*}{$M$} & IULIUS & TERTIUS & B & & & NO-AU-KA-KLA-Tanzenberg & I-MII & CIL-1II-4932 \\
\hline & IULIUS & TITUS & $\mathrm{R}$ & $\mathrm{P}$ & & NO-AU-ST-LIE-Wörschach & $\mid \cdot \mathrm{Cl}$ & CIL-III-5644 \\
\hline & IULIUS & TUTOR & $\mathrm{R}$ & $\mathrm{P}$ & & NO-AU-KA-KLA-St.Veit & i & CIL-III-4845 \\
\hline \multirow[t]{4}{*}{ TIB. } & IULIUS & URSULUS & $\mathrm{R}$ & i & DEUS SOL INVICTUS & NO-AU-OBO-Linz & IV & AE $56-82$ \\
\hline & IULIUS & VALENTINUS 1 & $\mathrm{R}$ & $\mathrm{E}$ & & NO-AL-BA-TR-Chieming & $\|-\|$ & CIL-III-11772 \\
\hline & IULIUS & VALENTINUS 2 & $\mathrm{R}$ & 1 & & NO-AU-ST-LE-Seggau & CIII & IRS-149 \\
\hline & IULIUS & VENANTIUS & $\mathrm{R}$ & & & NO-AU-KA-KLA-Pölling & $\mathrm{FI}-1 \mathrm{I}$ & CLL-1II-11580 \\
\hline c. & IULIUS & VEPO & $i$ & 1 & & NO-YU-SL-Celle & 1 & CIL-111-5232 \\
\hline c. & IULFUS & VERECUNDUS & A & & C/BUS? AUGUSTUS & NO-AU-OS-LI-Görtschach & $\mid-11$ & CIL-III-4721 \\
\hline \multirow[t]{4}{*}{ L. } & IULIUS & VERECUNDUS & $\mathrm{R}$ & & & NO-AU-OBO-WEL-St.Bernhardin & $|-1|$ & CIL-117-11788 \\
\hline & IUEIUS & VIBIANUS & $\mathrm{R}$ & & FORTUNAAUGUSTA & NO-AU-KA-KLA-Töltschach & FII-CIII & CIL-1II- 4778 \\
\hline & IULIUS & VICTOR & $\mathrm{R}$ & & & NO-AL-BA-RO-Eggstătt & FII & CIL-III-5567 \\
\hline & IULIUS & VICTORINus & R & & DII MANES & NO-AL-BA-RO-Eggstätt & FII & ClL-III-5567 \\
\hline \multirow[t]{2}{*}{ G. } & IULIUS & VOGITOUTUS & $i$ & $\rho$ & & NO-AU-KA-KLA-Tóltschach & 1 & CIL-III-4908 \\
\hline & IULIUS & APRICIUS & & $i$ & & NO-AU-OBO-EN-Lorch & FII-III & CIL-III-5671 \\
\hline \multirow[t]{24}{*}{ C. } & IULIUS & CENSONIUS & & L? & & NO-AU-KA-KLA-Zollfeld & 1 & CIL-III-4923 \\
\hline & IULIUS-A 1 & & & P? & & NO-AU-KA-WO-St.Michael & $|-| \mid$ & CIL-1II-5090 \\
\hline & IULIUS-A? & & & $\mathrm{P} ?$ & & NO-AU-KA-VIL-Saifnitz & FIIII & CIL-1II-4717 \\
\hline & IULIUS.A 3 & & & & & NO-AU-KA-KLA-Zolifeld & MIIIII & CIL $-1 \mid 1-4819$ \\
\hline & FULIUS-A 4 & & & $P ?$ & & NO-YU-SL-St,Lambrecht & II & IRS-277 \\
\hline & IULIUS-A 5 & & & & & NO-AU-KA-KLA-Zollfeld & i & Cll--1I-4923 \\
\hline & $\begin{array}{l}\text { IULIUS-A } 6 \\
\text { INA }\end{array}$ & & & $p ?$ & & NO-AU-KA-KLA-Untermühlbach & $|-| \mid$ & CIL-\|II-11560 \\
\hline & IULIUS-A 7 & & & $P ?$ & & NO-YU-SL-Celjo & i & CIL-III-5227 \\
\hline & IULIUS-A B & & & $P ?$ & & NO-AU-ST-LE-Seggau & i & CHL-III-5367 \\
\hline & IULIUS:A9 9 & & & $\mathrm{P} ?$ & & NO-AU-KA-KLA-Arndorf & $\|\| \|$, & ClL_-11|-4816 \\
\hline & IULIUS-A 10 & & & $\mathrm{P} ?$ & & NO-AU-KA-KLA-Arndorf & $\|-1\|$ & CIL. $-\| \mid=4816$ \\
\hline & IULIUS-A 11 & & & $\mathrm{P}$ & & NO-AU-ST-GR-Waldstein & 1 & CIL--1II-4925 \\
\hline & IULIUS? 1 & & & $\mathrm{P}$ & & NO-AU-ST-LE-Kaindorf & |-111 & ClL- $-111-5400$ \\
\hline & IULIUS? 2 & & & L & DII MANES & NO-AU-ST-GR-Waldstein & I & CIL-III-5453 \\
\hline & $\begin{array}{l}\text { IULIUS? } \\
\text { IUt }\end{array}$ & ADIUTOR & $\mathrm{R}$ & & & NO-AU-KA-KLA-St.Velt & 1 & CIL-III-4925 \\
\hline & IULIUS? & AQUILINUS & $\mathrm{R}$ & & & NO-AU-SA-Salzburg & MII-M\|II & ClL-1II-5548 \\
\hline & IULIUS? & BARDUS & i & & & NO-AU-KA-KLA-Feistritz & $\mathrm{Fl}+11$ & CLL-1II-4838 \\
\hline & IULIUS? & FRONTO & $\mathrm{R}$ & & & NO-YU-SL-CE-Gonobitz & $\mathrm{M} \cdot \mathrm{FI}$ & CIL-III-15205. \\
\hline & IULIUS? & GIAMILLUS & $i$ & & & NO-AU-KA-KLA-S.Georgen am Längsee & । & CIL-IIJ-11554 \\
\hline & IULIUS? & MARTIALIS & $R$ & & & NO-AU-KA-KLA-Magdalensberg & & CIL-III-4962A \\
\hline & IULIUS? & PRIMUS & $\mathrm{R}$ & & & NO-YU-SL-MAR-Tainach am Pachom(?) & $|-|||$ & CIL-III-11716 \\
\hline & IULIUS? & ROMULUS & $\mathrm{R}$ & & & NO-YU-SL-SB-Poljcane & 11-III & CIL-1II-5299 \\
\hline & IULIUS? & SECUNDINUS & $\mathrm{R}$ & & & NO-AU-ST-LE-Landscha & FII-III & GIL-111-5362 \\
\hline & IULIUS? & SURIANUS & 1 & & & NO-YU-SL-SB-Poljcane & $\| 1 \mathrm{III}$ & CIL-III-5299 \\
\hline
\end{tabular}

Tabla 3. Iulii en Noricum.

\begin{tabular}{|c|c|c|c|c|c|}
\hline \multirow[t]{2}{*}{ PRAE } & NOMEN & COGNOMEN & ETN & STA & DIVINIDAD \\
\hline & ANTONIUS & IULIANUS & $\mathrm{R}$ & & IUPPITER OPTIM. MAX. \\
\hline \multirow[t]{3}{*}{ c. } & ATILIUS & IULIANUS & $\mathbf{R}$ & & \\
\hline & CELERIUS & IULIANUS & $\mathrm{R}$ & & IUPPITER OPTIM. MAX. \\
\hline & CUPITIA & IULIANA & $\mathrm{R}$, & & DII MANES \\
\hline c. & IULIA & IULIANA & $\mathrm{R}$ & & CBUS?AUGUSTUS \\
\hline TIB. & IULIUS & IULIANUS & $\mathrm{R}$ & & DIIMANES \\
\hline Q. & MARIUS & IULIANUS & $\mathrm{R}$ & D.1 & \\
\hline Q. & POMPEIUS & IULIANUS & $\mathrm{R}$ & & \\
\hline \multirow[t]{8}{*}{ L. } & SEPTIMIUS & IULIANUS & $\mathrm{R}$ & $P$ & DIIMANES \\
\hline & SPERATIA & IULIANA & $\mathrm{R}$ & & \\
\hline & ULPIA & IULIANA & $\mathrm{R}$ & & IUPPITER DOLICHENUS \\
\hline & & IULIANA & $\mathrm{R}$ & $\mathrm{s}$ & \\
\hline & & IULIANNA & $\mathrm{R}$ & & \\
\hline & & IULIANUS 1 & $\mathrm{R}$ & & \\
\hline & & IULIANUS? & $\mathrm{R}$ & & \\
\hline & & IULIANUS 3 & $\mathrm{R}$ & & \\
\hline
\end{tabular}

LUGARHALLA

NO-YU-SL-TRB-Trojane

NO-YU-SL-TIT-Sempe

NO-AU-ST-LE-Segga

NO-AU-OS-LI-Görtschach

NO-AU-KA-KLA-Zollfeld

NO-YU-SL-Celje

NO-AU-ST-LE-Seggau

NO-YU-SL-Celje

NO-AU-NI-STP-Mauer an der Url

NO-YU-ST-Cel

NO-YU-SL-Celje

NO-AU-ST.LE-Rottenman

NO-AU-KA-KLA-S. Donat

NO-YU-SL-MAR-Smartno na Pohoriu
CRONO BIBLIO

CIL-III-5120

FI-II UII CLL-III-5115

FII-III CIL-III-522

CIL-III-4721

CIL.III-4929

CIL-AII-5254

FII-CIII CIL-111-5563

-CIII CIL-III-5240

AE39-270

MII CIL-III-5254

CIII CIL-III-5637

CIL-III-4999

CII CIL-III-5027

Tabla 4. Cognomina derivados del nomen Iulius en Noricum. 


\section{BIBLIOGRAFIA}

ALFÖLDY, G. 1969, "Epigraphica Norica", Epigraphische Studien, 8, 1-34.

ALFÖLDY, G. 1970, "Patrimonium regni Norici", Bonner Jahrbücher, 170, 163-177.

ANNÉE ÉPIGRAPHIQUE, 1900-1994, París.

CARRIE, J.M. 1986, "L'esercito: transformazioni funzionali ed economie locali",en A. Giardina, Societá romana e impero tardonatico l: Instituzione ceti, economie, Roma, 449-779.

CHARLESWORTH, M.P. 1976 (Ed. 1926), Trade routes and commerce of the Roman Empire, Chicago.

CHRISTOL, M. 1988, "Armées et societé politique dans l'Empire romain au IIle siècle ap. J.C.", Civilità Classica e Cristiana, 9,2, 169-204.

CORPUS INSCRIPTIONUM LATINARUM, vol. III y suppl.

CRESPO, S. 1992, "El término alumnus indicador de dependencia personal en Hispania romana", Minerva, 225-239.

D'ARMS, J.H. 1981, Commerce and Social Standing in Ancient Rome, Cambridge.

DEVIJVER, H. 1976/77, Prosopographia Militiarum equestrium quae fuerunt ab Augusto ad Gallienum, Vol. I, Louvain.

DRUMMOND, S.K. y NELSON, L.H. 1994, The Western Frontiers of Imperial Rome, New York.

DUSANIC, S. 1977, "Aspects of Roman Mining in Noricum, Pannonia, Dalmatia and Moesia Superior", Aufstieg und Niedergang der römischen Welt, 6, 52-94.

EGGER, E. 1961, Die Stadt auf dem Magdalensberg ein Grosshan delsplatz, Österreichischen Akademie der Wissenschaften, Wien.

FORNI, G. 1953, // reclutamento delle legioni da Augusto à Diocleziano, Roma.

GALLEGO FRANCO, H. 1996, Prosopografía, Onomástica y Sociedad en el Imperio Romano. Las provincias de Rhaetia, Noricum y Pannonia Superior e Inferior, Valladolid, (microficha).

GALLEGO FRANCO, H. 1998, Las Estructuras Sociales del Imperio Romano. Las provincias de Rhaetia, Noricum y Pannonia, Valladolid.

GARNSEY, P., HOPKINS, K. y WHITTAKER, C.R. 1989, Trade in the Ancient Economy, London.

GIARDINA, A. 1991, El hombre romano, Madrid.

HARDING, M. y JACOBSEN, G. 1989, "Norditalische Zuwanderung nach Celeia während der ersten zwei Jahrhunderte n. chr.", Acta Archaeologica Academiae Scientiarum Hungaricae, XLI, 227-232.

HENZEN, G., ROSSI, I.B., MOMMSEM, TH. y WILMANNS, G. 1875 y 1881, Ephemeris epigraphica, vol. II y IV, Berlin.

HOLDER, A. 1961-1962, Alt-Celtischer Sprachschatz, vols. I-II-III, Graz.

KAJANTO, I. 1965, The Latin Cognomina, Helsinki.

KAJANTO, I. 1977, "On the peculiarities of women's nomenclature", L'Onomastique Latine, Actes du Colloque International, 13-15 oct.París, 1975, París, 147-158.

KELLNER, H.J. 1976, "Augsburg, Provinz- hauptstadt Raetiens", Aufstieg und Niedergang der römischen Welt, II,5.2, 690-717.
LUTTWAK, E.N. 1976, The Grand Strategy of the Roman Empire, London.

MARTIN, J.P. 1990, Les provinces romaines d'Europe Centrale et Occidentale, París.

MARTIN, J.P. 1991, Les provinces romaines d'Europe Centrale et Occidentale. Société et Religions, Paris.

PASTOR MUÑOZ, M. y MENDOZA EGUARAS, E. 1987 , Inscripciones latinas de la provincia de Granada, Granada.

PFLAUM, H.G. 1960-1961, Les carrières procuratoriennes équestres sous le Haut-Empire romain, vol.l, París.

SASEL, J. 1954, "C. Iulius Vepo", Ziva Antika, IV, 346363.

SASEL, A. \& J. 1978, "Inscriptiones Latinae quae in Iuguslavia inter annos MCMLX et MCMLXX repertae et editae sunt", Situla, XIX

SASEL, A. \& J. 1963, "Inscriptiones Latinae quae in luguslavia inter annos MCMXL et MCMLX repertae et editae sunt", Situla, $V$.

SASEL, A. \& J. 1986, "Inscriptiones latinae quae in Iuguslavia inter annos MCMII et MCMXL repertae et editae sunt", Situla, XXV.

SCHLIPPSCHUH, O. 1974, Die Händler in römischen Kaiserreich in Gallien, Germanien und den donauprovinzen Raetien, Noricum und Pannonien, Amsterdam.

SERRANO DELGADO, J.M. 1988, Status y promoción social de los libertos en España romana, Sevilla.

SOLIN, H. 1971, Beitrage zur kenntnis der Griechischen Personennamen in Rom, Helsinki.

SOLIN, H. 1982, Griech Personennamen in Rom, Berlin

SOLIN, H. y SALOMIES, O. 1988, Repertorium nominum gentilium et cognominum Latinorum, Olms.

ULBERT, G. 1971, "Zur Grenze zwischen den römischen Provinzen Norikum und Raetien", Bayerische Vorgeschichtsblätter, 36, 101-123.

VOLLMER, FR. 1915, Inscriptiones Baviariae Romanae sive Inscriptiones provinciae Raetiae, adiectis aliquot Noricis Italicisque, München.

WACHER, J. 1987, The Roman Empire, London.

WAGNER, F. 1956-1957 [1958], "Neue Inschriften aus Raetien. Nachträge zu Fr. Vollmer, Inscriptiones Baivariae Romanae", Bericht der Römisch-Germanischen Komission, XXXVII-XXXVIII, 215-264.

WALSER, G. 1983, Die römischen Strassen und Meilensteine in Raetien, Stuttgart.

WEAVER, P.R.C. 1972, Familia Caesaris. A social stu-dy of the Emperor's freedmen and slaves, Cambridge.

WEBER, E. 1969, Die römerzeitlichen Inschriften der Steiermark, Graz.

WHITTAKER, C.R. 1989, Les Frontières de l'Empire romain, París.

WHITTAKER, C.R. 1994, Frontiers of the Roman Empire. A Social and Economic Study, London.

ZAJAC, J. 1990-1992, "Il cognomina dei patroni", Acta Antiqua Academiae Scientiarum Hungaricae, 33, 350359. 